Advances in Dynamical Systems and Applications (ADSA).

ISSN 0973-5321, Volume 15, Number 2, (2020) pp. 231-247

(C) Research India Publications

https://dx.doi.org/10.37622/ADSA/15.2.2020.231-247

\title{
Existence of a Weak Solution to the Maxwell-Stokes Type Equation by the Penalty Method
}

\author{
Junichi Aramaki \\ Division of Science, Faculty of Science and Engineering, \\ Tokyo Denki University, Hatoyama-machi, Saitama 350-0394, Japan.
}

\begin{abstract}
In this paper, we show the existence of a weak solution to the Maxwell-Stokes type equation by the penalty method introduced by Temam. Our approximate equation is nonlinear and contains so called $p$-curl system. Furthermore, we obtain the continuous dependence of the weak solution on the data.
\end{abstract}

Keywords: Maxwell-Stokes type equation, weak solution, penalty method, minimization problem

2010 Mathematics Subject Classifications: 35A05, 35H30, 35A15, 35D05

\section{INTRODUCTION}

In this paper, we show the existence of a weak solution to the Maxwell-Stokes type equation by the penalty method introduced by Temam [9] (cf. Dautray and Lions [6, vol. 7] or Girault and Raviart [7]).

More precisely, we consider the following Stokes problem in a bounded, Lipschitz-continuous domain $\Omega \subset \mathbb{R}^{d}$ with boundary $\Gamma$.

$$
\begin{cases}-\Delta \boldsymbol{u}+\nabla \pi=\boldsymbol{f} & \text { in } \Omega, \\ \operatorname{div} \boldsymbol{u}=0 & \text { in } \Omega, \\ \boldsymbol{u}=\mathbf{0} & \text { on } \Gamma .\end{cases}
$$

The penalty method replaces the Stokes problem (1.1) by

$$
\begin{cases}-\Delta \boldsymbol{u}_{\varepsilon}-\frac{1}{\varepsilon} \nabla \operatorname{div} \boldsymbol{u}_{\varepsilon}=\boldsymbol{f} & \text { in } \Omega \\ \boldsymbol{u}_{\varepsilon}=\mathbf{0} & \text { on } \Gamma\end{cases}
$$

where $\varepsilon$ is a positive parameter that will tend to zero. The pressure $\pi$ is approximated by 
$\pi_{\varepsilon}=-\frac{1}{\varepsilon} \operatorname{div} \boldsymbol{u}_{\varepsilon}$, and $\boldsymbol{u}_{\varepsilon}$ approximates $\boldsymbol{u}$. Since the problem (1.2) is the Dirichlet problem for the elliptic equation, the problem (1.2) has a unique solution $\boldsymbol{u}_{\varepsilon}$. Temam established the convergence of $\left(\boldsymbol{u}_{\varepsilon}, \pi_{\varepsilon}\right)$ to $(\boldsymbol{u}, \pi)$, and more precisely, proved the following theorem.

Theorem 1.1 (Temam). Let $\Omega$ be a bounded, Lipschitz-continuous domain in $\mathbb{R}^{d}$, and $\boldsymbol{f} \in \boldsymbol{H}^{-1}(\Omega)$. Then, as $\varepsilon \rightarrow 0$, we have

$$
\boldsymbol{u}_{\varepsilon} \rightarrow \boldsymbol{u} \text { in } \boldsymbol{H}_{0}^{1}(\Omega) \text { and } \pi_{\varepsilon} \rightarrow \pi \text { in } L^{2}(\Omega),
$$

where $(\boldsymbol{u}, \pi)$ is the solution of the homogeneous Stokes problem (1.1).

Amrouche and Girault [1] extended this convergence to $\boldsymbol{W}^{m, p}(\Omega)$.

In the present paper, we shall show that the penalty method can be applicable to the Maxwell-Stokes problem in the case $d=3$. In order to do so, we replace $-\Delta \boldsymbol{u}$ in the first equatin of (1.1) with a nonlinear term as in the following system.

$$
\begin{cases}\operatorname{curl}\left[S_{t}\left(x,|\operatorname{curl} \boldsymbol{u}|^{2}\right) \operatorname{curl} \boldsymbol{u}\right]+\nabla \pi=\boldsymbol{f} & \text { in } \Omega, \\ \operatorname{div} \boldsymbol{u}=0 & \text { in } \Omega, \\ \boldsymbol{u}=\mathbf{0} & \text { on } \Gamma,\end{cases}
$$

where the function $S(x, t)$ is a Carathéodory function in $\Omega \times[0, \infty)$ satisfying some structure conditions. The first equation of (1.3) contains so called $p$-curl system

$$
\operatorname{curl}\left[|\operatorname{curl} \boldsymbol{u}|^{p-2} \operatorname{curl} \boldsymbol{u}\right]+\nabla \pi=\boldsymbol{f}
$$

in a special case. The equation (1.3) is nonlinear, and when $p>2$, it is degenerate and when $1<p<2$, it is singular. In a special case of $S(x, t)$ and $p=2$, (1.3) reduces to the equation (1.1), so our result extends Theorem 1.1. Our approximate system is as follows.

$$
\begin{cases}\operatorname{curl}\left[S_{t}\left(x,\left|\operatorname{curl} \boldsymbol{u}_{\varepsilon}\right|^{2}\right) \operatorname{curl} \boldsymbol{u}_{\varepsilon}\right]-\frac{1}{\varepsilon} \nabla\left[S_{t}\left(x,\left(\operatorname{div} \boldsymbol{u}_{\varepsilon}\right)^{2}\right) \operatorname{div} \boldsymbol{u}_{\varepsilon}\right]=\boldsymbol{f} & \text { in } \Omega \\ \boldsymbol{u}_{\varepsilon}=\mathbf{0} & \text { on } \Gamma .\end{cases}
$$

Since this equation is nonlinear, it is necessary to investigate this problem from a different point of view. Then we show that

$$
\boldsymbol{u}_{\varepsilon} \rightarrow \boldsymbol{u} \text { in } \boldsymbol{W}_{0}^{1, p}(\Omega) \text { and } \pi_{\varepsilon}:=-\frac{1}{\varepsilon} S_{t}\left(x,\left(\operatorname{div} \boldsymbol{u}_{\varepsilon}\right)^{2}\right) \operatorname{div} \boldsymbol{u}_{\varepsilon} \rightarrow \pi \text { in } L^{p}(\Omega),
$$

where $(\boldsymbol{u}, \pi)$ is a weak solution of (1.3).

The paper is organized as follows. In section 2, we give some preliminaries and the main theorem that states the existence of the weak solution to the problem (1.3). Section 3 is devoted the proof of the main theorem. In section 4 , we show the continuous dependence of the solution on the data. 


\section{PRELIMINARIES AND THE MAIN THEOREM}

This section consists of two subsections. In subsection 2.1, we give some preliminaries that are necessary later. In subsection 2.2, we give the notion of a weak solution for the Maxwell-Stokes problem and state the main theorem.

\subsection{Preliminaries}

Let $\Omega$ be a bounded domain in $\mathbb{R}^{3}$ with a Lipshitz-continuous boundary $\Gamma, 1<p<\infty$ and let $p^{\prime}$ be the conjugate exponent i.e., $(1 / p)+\left(1 / p^{\prime}\right)=1$. From now on we use $L^{p}(\Omega)$ and $W_{0}^{1, p}(\Omega)$ for the standard $L^{p}$ and Sobolev spaces of functions. For any Banach space $B$, we denote $B \times B \times B$ by boldface character $\boldsymbol{B}$. Hereafter, we use this character to denote vector and vector-valued functions, and we denote the standard Euclidean inner product of vectors $\boldsymbol{a}$ and $\boldsymbol{b}$ in $\mathbb{R}^{3}$ by $\boldsymbol{a} \cdot \boldsymbol{b}$.

We assume that a Carathéodory function $S(x, t)$ in $\Omega \times[0, \infty)$ satisfies the following structure conditions. For a.e. $x \in \Omega, S(x, t) \in C^{2}((0, \infty)) \cap C^{0}([0, \infty))$, and there exist $1<p<\infty$ and positive constants $0<\lambda \leq \Lambda<\infty$ such that for a.e. $x \in \Omega$,

$$
\begin{array}{r}
S(x, 0)=0 \text { and } \lambda t^{(p-2) / 2} \leq S_{t}(x, t) \leq \Lambda t^{(p-2) / 2} \text { for } t>0, \\
\lambda t^{(p-2) / 2} \leq S_{t}(x, t)+2 t S_{t t}(x, t) \leq \Lambda t^{(p-2) / 2} \text { for } t>0, \\
\text { If } 1<p<2, S_{t t}(x, t)<0 \text {, and if } p \geq 2, S_{t t}(x, t) \geq 0 \text { for } t>0 .
\end{array}
$$

We note that from (2.1a), we have

$$
\frac{2}{p} \lambda t^{p / 2} \leq S(x, t) \leq \frac{2}{p} \Lambda t^{p / 2} \text { for } t \geq 0
$$

Example 2.1. If $S(x, t)=\nu(x) t^{p / 2}$, where $\nu$ is a measurable function in $\Omega$ and satisfies $0<\nu_{*} \leq \nu(x) \leq \nu^{*}<\infty$ for a.e. in $\Omega$ for some constants $\nu_{*}$ and $\nu^{*}$, then it follows from elementary calculations that (2.1a)-(2.1c) hold. .

We give a monotonicity lemma.

Lemma 2.2. (i) There exists a constant $c_{1}>0$ such that for all $t, s \in \mathbb{R}$,

$$
\left(S_{t}\left(x, t^{2}\right) t-S_{t}\left(x, s^{2}\right) s\right)(t-s) \geq \begin{cases}c_{1}|t-s|^{p} & \text { if } p>2, \\ c_{1}(|t|+|s|)^{p-2}|t-s|^{2} & \text { if } 1<p \leq 2 .\end{cases}
$$


(ii) There exists a constant $c_{2}>0$ such that for all $\boldsymbol{a}, \boldsymbol{b} \in \mathbb{R}^{3}$,

$$
\begin{aligned}
& \left(S_{t}\left(x,|\boldsymbol{a}|^{2}\right) \boldsymbol{a}-S_{t}\left(x,|\boldsymbol{b}|^{2}\right) \boldsymbol{b}\right) \cdot(\boldsymbol{a}-\boldsymbol{b}) \\
& \geq \begin{cases}c_{2}|\boldsymbol{a}-\boldsymbol{b}|^{p} & \text { if } p>2, \\
c_{2}(|\boldsymbol{a}|+|\boldsymbol{b}|)^{p-2}|\boldsymbol{a}-\boldsymbol{b}|^{2} & \text { if } 1<p \leq 2 .\end{cases}
\end{aligned}
$$

Proof. We prove (i). If we put $I(x, t)=\left(S_{t}\left(x, t^{2}\right) t-S_{t}\left(x, s^{2}\right) s\right)(t-s)$, then we can write

$$
\begin{aligned}
I(x, t)= & \int_{0}^{1} \frac{d}{d \theta}\left[S_{t}\left(x,(\theta t+(1-\theta) s)^{2}\right)(\theta t+(1-\theta) s)\right] d \theta(t-s) \\
= & \int_{0}^{1}\left\{S_{t}\left(x,(\theta t+(1-\theta) s)^{2}\right)\right. \\
& \left.\quad+2 S_{t t}\left(x,(\theta t+(1-\theta) s)^{2}\right)(\theta t+(1-\theta) s)^{2}\right\} d \theta(t-s)^{2} .
\end{aligned}
$$

From (2.1b), we have

$$
I(x, t) \geq \lambda \int_{0}^{1}|\theta t+(1-\theta) s|^{p-2} d \theta|t-s|^{2} .
$$

When $1<p \leq 2$, it is trivial that

$$
I(x, t) \geq \lambda(|t|+|s|)^{p-2}|t-s|^{2} .
$$

We consider the case $p>2$. If $|t| \geq|t-s|$, then

$$
|\theta t+(1-\theta) s|=|t+(1-\theta)(s-t)| \geq|t|-(1-\theta)|s-t| \geq \theta|t-s| .
$$

Thus it follows from (2.3) that

$$
I(x, t) \geq \lambda \int_{0}^{1} \theta^{p-2} d \theta|t-s|^{p}=\frac{\lambda}{p-1}|t-s|^{p} .
$$

If $|t|<|t-s|$, we write

$$
\int_{0}^{1}|\theta t+(1-\theta) s|^{p-2} d \theta=\int_{0}^{1} \frac{\left(|\theta t+(1-\theta) s|^{2}\right)^{p / 2}}{|\theta t+(1-\theta) s|^{2}} d \theta .
$$

Since

$$
\begin{aligned}
|\theta t+(1-\theta) s|^{2} & =|t+(1-\theta)(s-t)|^{2} \\
& \leq(|t|+(1-\theta)|s-t|)^{2} \\
& \leq(|s-t|+(1-\theta)|s-t|)^{2} \\
& =(2-\theta)^{2}|s-t|^{2} \\
& \leq 4|s-t|^{2}
\end{aligned}
$$


for $0 \leq \theta \leq 1$, using the Jensen inequality (cf. Jost [8, p. 122]), we have

$$
\begin{aligned}
I(x, t) & \geq \frac{\lambda}{4} \int_{0}^{1}\left(|t+(1-\theta)(s-t)|^{2}\right)^{p / 2} d \theta \\
& \geq \frac{\lambda}{4}\left(\int_{0}^{1}(t+(1-\theta)(s-t))^{2}\right)^{p / 2} \\
& \geq \frac{\lambda}{4} \frac{1}{3^{p / 2}}\left(t^{2}+s^{2}+t s\right)^{p / 2} \\
& \geq \frac{\lambda}{4} \frac{1}{3^{p / 2}} \frac{1}{4^{p / 2}}|t-s|^{p} .
\end{aligned}
$$

For the proof of (ii), see Aramaki [5, Lemma 3.6].

Lemma 2.3. There exists a constants $C_{1}>0$ depending only on $\Lambda$ and $p$ such that for any $\boldsymbol{a}, \boldsymbol{b} \in \mathbb{R}^{3}$,

$$
\begin{aligned}
& \left|S_{t}\left(x, s,|\boldsymbol{a}|^{2}\right) \boldsymbol{a}-S_{t}\left(x, s,|\boldsymbol{b}|^{2}\right) \boldsymbol{b}\right| \\
& \leq \begin{cases}C_{1}|\boldsymbol{a}-\boldsymbol{b}|^{p-1} & \text { if } 1<p<2, \\
C_{1}(|\boldsymbol{a}|+|\boldsymbol{b}|)^{p-2}|\boldsymbol{a}-\boldsymbol{b}| & \text { if } p \geq 2\end{cases}
\end{aligned}
$$

For the proof, see Aramaki [3].

\subsection{The main theorem}

We consider the following Maxwell-Stokes system.

$$
\begin{cases}\operatorname{curl}\left[S_{t}\left(x,|\operatorname{curl} \boldsymbol{u}|^{2}\right) \operatorname{curl} \boldsymbol{u}\right]+\nabla \pi=\boldsymbol{f} & \text { in } \Omega, \\ \operatorname{div} \boldsymbol{u}=0 & \text { in } \Omega \\ \boldsymbol{u}=\mathbf{0} & \text { on } \Gamma\end{cases}
$$

Definition 2.4. Let $\boldsymbol{f} \in \boldsymbol{W}^{-1, p^{\prime}}(\Omega)$ that is the dual space of $\boldsymbol{W}_{0}^{1, p}(\Omega)$. We say $(\boldsymbol{u}, \pi) \in \boldsymbol{W}_{0}^{1, p}(\Omega) \times L^{p^{\prime}}(\Omega) / \mathbb{R}$ is a weak solution of (2.4), if $\boldsymbol{u}$ is divergence free and $(\boldsymbol{u}, \pi)$ satisfies

$$
\int_{\Omega} S_{t}\left(x,|\operatorname{curl} \boldsymbol{u}|^{2}\right) \operatorname{curl} \boldsymbol{u} \cdot \operatorname{curl} \boldsymbol{v} d x-\int_{\Omega} \pi \operatorname{div} \boldsymbol{v} d x=\langle\boldsymbol{f}, \boldsymbol{v}\rangle
$$

for all $\boldsymbol{v} \in \boldsymbol{W}_{0}^{1, p}(\Omega)$, where $\langle\boldsymbol{f}, \boldsymbol{v}\rangle$ denotes the duality between $\boldsymbol{f} \in \boldsymbol{W}^{-1, p^{\prime}}(\Omega)$ and $\boldsymbol{v} \in \boldsymbol{W}_{0}^{1, p}(\Omega)$.

We are in a position to state the main theorem. 
Theorem 2.5. Let $\Omega \subset \mathbb{R}^{3}$ be a bounded, Lipcshitz-continuous domain in $\mathbb{R}^{3}$, and assume that a Carathéodory function $S(x, t)$ satisfies the structure conditions (2.1a)-(2.1c). Then for any $\boldsymbol{f} \in \boldsymbol{W}^{-1, p^{\prime}}(\Omega)$, the Maxwell-Stokes system (2.4) has a unique weak solution $(\boldsymbol{u}, \pi) \in \boldsymbol{W}_{0}^{1, p}(\Omega) \times L^{p^{\prime}}(\Omega) / \mathbb{R}$, and there exists a constant $C>0$ depending only on $p, \lambda, \Lambda$ and $\Omega$ such that

$$
\|\boldsymbol{u}\|_{\boldsymbol{W}_{0}^{1, p}(\Omega)}^{p}+\|\pi\|_{L^{p^{\prime}}(\Omega) / \mathbb{R}}^{p^{\prime}} \leq C\|\boldsymbol{f}\|_{\boldsymbol{W}^{-1, p^{\prime}}(\Omega)}^{p^{\prime}} .
$$

\section{PROOF OF THEOREM 2.5}

In this section, we prove Theorem 2.5 by the penalty method. In order to do so, let $0<\varepsilon \leq 1$. We consider the following system, and give the notion of its weak solution.

$$
\begin{cases}\operatorname{curl}\left[S_{t}\left(x,|\operatorname{curl} \boldsymbol{u}|^{2}\right) \operatorname{curl} \boldsymbol{u}\right]-\frac{1}{\varepsilon} \nabla\left[S_{t}\left(x,(\operatorname{div} \boldsymbol{u})^{2}\right) \operatorname{div} \boldsymbol{u}\right]=\boldsymbol{f} & \text { in } \Omega, \\ \boldsymbol{u}=\mathbf{0} & \text { on } \Gamma\end{cases}
$$

Definition 3.1. We say that $\boldsymbol{u}_{\varepsilon} \in \boldsymbol{W}_{0}^{1, p}(\Omega)$ is a weak solution of (3.1), if $\boldsymbol{u}_{\varepsilon}$ satisfies

$$
\begin{array}{r}
\int_{\Omega}\left\{S_{t}\left(x,\left|\operatorname{curl} \boldsymbol{u}_{\varepsilon}\right|^{2}\right) \operatorname{curl} \boldsymbol{u}_{\varepsilon} \cdot \operatorname{curl} \boldsymbol{v}+\frac{1}{\varepsilon} S_{t}\left(x,\left(\operatorname{div} \boldsymbol{u}_{\varepsilon}\right)^{2}\right)\left(\operatorname{div} \boldsymbol{u}_{\varepsilon}\right)(\operatorname{div} \boldsymbol{v})\right\} d x \\
=\langle\boldsymbol{f}, \boldsymbol{v}\rangle \text { for all } \boldsymbol{v} \in \boldsymbol{W}_{0}^{1, p}(\Omega) .
\end{array}
$$

For any fixed $0<\varepsilon \leq 1$, define a functional

$$
E_{\varepsilon}[\boldsymbol{v}]=\frac{1}{2} \int_{\Omega}\left\{S\left(x,|\operatorname{curl} \boldsymbol{v}|^{2}\right)+\frac{1}{\varepsilon} S\left(x,(\operatorname{div} \boldsymbol{v})^{2}\right)\right\} d x-\langle\boldsymbol{f}, \boldsymbol{v}\rangle .
$$

We consider the following minimization problem: to find $\boldsymbol{u}_{\varepsilon} \in \boldsymbol{W}_{0}^{1, p}(\Omega)$ such that

$$
E_{\varepsilon}\left[\boldsymbol{u}_{\varepsilon}\right]=\alpha:=\inf _{\boldsymbol{v} \in \boldsymbol{W}_{0}^{1, p}(\Omega)} E_{\varepsilon}[\boldsymbol{v}]
$$

We call such a $\boldsymbol{u}_{\varepsilon}$ a minimizer of $\alpha$. Then we have the following proposition.

Proposition 3.2. Let $0<\varepsilon \leq 1$ and $\boldsymbol{f} \in \boldsymbol{W}^{-1, p^{\prime}}(\Omega)$. Then the minimization problem (3.3) has a minimizer $\boldsymbol{u}_{\varepsilon} \in \boldsymbol{W}_{0}^{1, p}(\Omega)$.

Proof. According to Amrouche and Seloula [2], we first note that there exists a constant $C>0$ depending only on $p$ and $\Omega$ such that

$$
\|\nabla \boldsymbol{v}\|_{\boldsymbol{L}^{p}(\Omega)} \leq C\left(\|\operatorname{curl} \boldsymbol{v}\|_{\boldsymbol{L}^{p}(\Omega)}+\|\operatorname{div} \boldsymbol{v}\|_{L^{p}(\Omega)}\right)
$$


for all $\boldsymbol{v} \in \boldsymbol{W}_{0}^{1, p}(\Omega)$, and from the Poincare inequality, there exists a constant $c>0$ depending only on $p$ and $\Omega$ such that

$$
\|\boldsymbol{v}\|_{\boldsymbol{L}^{p}(\Omega)} \leq c\|\nabla \boldsymbol{v}\|_{\boldsymbol{L}^{p}(\Omega)} \text { for all } \boldsymbol{v} \in \boldsymbol{W}_{0}^{1, p}(\Omega) .
$$

Thus there exists a constant $C_{2}>0$ depending only on $p$ and $\Omega$ such that

$$
\|\boldsymbol{v}\|_{\boldsymbol{W}_{0}^{1, p}(\Omega)}^{p} \leq C_{2}\left(\|\operatorname{curl} \boldsymbol{v}\|_{\boldsymbol{L}^{p}(\Omega)}^{p}+\|\operatorname{div} \boldsymbol{v}\|_{L^{p}(\Omega)}^{p}\right) .
$$

Since $0<\varepsilon \leq 1$, using (2.2), the Hölder inequality and the Young inequality, for any $\delta>0$ there exists a constant $C(\delta)>0$ such that

$$
\begin{aligned}
E_{\varepsilon}[\boldsymbol{v}] & \geq \frac{1}{2} \int_{\Omega}\left\{S\left(x,|\operatorname{curl} \boldsymbol{v}|^{2}\right)+S\left(x,(\operatorname{div} \boldsymbol{v})^{2}\right)\right\} d x-\langle\boldsymbol{f}, \boldsymbol{v}\rangle \\
& \geq \frac{\lambda}{p} \int_{\Omega}\left(|\operatorname{curl} \boldsymbol{v}|^{p}+|\operatorname{div} \boldsymbol{v}|^{p}\right) d x-\|\boldsymbol{f}\|_{\boldsymbol{W}^{-1, p^{\prime}}(\Omega)}\|\boldsymbol{v}\|_{\boldsymbol{W}_{0}^{1, p}(\Omega)} \\
& \geq \frac{\lambda}{p C_{2}}\|\boldsymbol{v}\|_{\boldsymbol{W}_{0}^{1, p}(\Omega)}^{p}-C(\delta)\|\boldsymbol{f}\|_{\boldsymbol{W}^{-1, p^{\prime}}(\Omega)}^{p^{\prime}}-\delta\|\boldsymbol{v}\|_{\boldsymbol{W}_{0}^{1, p}(\Omega)}^{p} .
\end{aligned}
$$

If we choose $\delta>0$ so that $\delta=\lambda / 2 p C_{2}$, then we have

$$
E_{\varepsilon}[\boldsymbol{v}] \geq \frac{\lambda}{2 p C_{2}}\|\boldsymbol{v}\|_{W_{0}^{1, p}(\Omega)}^{p}-C\left(\lambda / 2 p C_{2}\right)\|\boldsymbol{f}\|_{W^{-1, p^{\prime}}(\Omega)}^{p^{\prime}}>-\infty .
$$

Let $\left\{\boldsymbol{v}_{j}\right\} \subset \boldsymbol{W}_{0}^{1, p}(\Omega)$ be a minimizing sequence of $\alpha$, i.e.,

$$
E_{\varepsilon}\left[\boldsymbol{v}_{j}\right]=\alpha+o(1) \text { as } j \rightarrow \infty .
$$

From (3.5), $\left\{\boldsymbol{v}_{j}\right\}$ is bounded in $\boldsymbol{W}_{0}^{1, p}(\Omega)$. Since $\boldsymbol{W}_{0}^{1, p}(\Omega)$ is a reflexive Banach space, passing to a subsequence, we may assume that

$$
\boldsymbol{v}_{j} \rightarrow \boldsymbol{u}_{\varepsilon} \text { weakly in } \boldsymbol{W}_{0}^{1, p}(\Omega) \text { as } j \rightarrow \infty .
$$

According to Aramaki [4], we have

$$
\begin{gathered}
\int_{\Omega} S\left(x,\left|\operatorname{curl} \boldsymbol{u}_{\varepsilon}\right|^{2}\right) d x=\liminf _{j \rightarrow \infty} \int_{\Omega} S\left(x,\left|\operatorname{curl} \boldsymbol{v}_{j}\right|^{2}\right) d x, \\
\int_{\Omega} S\left(x,\left(\operatorname{div} \boldsymbol{u}_{\varepsilon}\right)^{2}\right) d x=\liminf _{j \rightarrow \infty} \int_{\Omega} S\left(x,\left(\operatorname{div} \boldsymbol{v}_{j}\right)^{2}\right) d x
\end{gathered}
$$

and clearly $\left\langle\boldsymbol{f}, \boldsymbol{v}_{j}\right\rangle \rightarrow\left\langle\boldsymbol{f}, \boldsymbol{u}_{\varepsilon}\right\rangle$ as $j \rightarrow \infty$. Therefore, we have

$$
E_{\varepsilon}\left[\boldsymbol{u}_{\varepsilon}\right] \leq \liminf _{j \rightarrow \infty} E_{\varepsilon}\left[\boldsymbol{v}_{j}\right]=\alpha
$$

So $\boldsymbol{u}_{\varepsilon}$ is a minimizer of $\alpha$. 
For any $\boldsymbol{v} \in \boldsymbol{W}_{0}^{1, p}(\Omega)$ and $t \in \mathbb{R}$, we have $E_{\varepsilon}\left[\boldsymbol{u}_{\varepsilon}\right] \leq E_{\varepsilon}\left[\boldsymbol{u}_{\varepsilon}+t \boldsymbol{v}\right]$. Thus, the Euler-Lagrange equation implies

$$
\begin{aligned}
\left.\frac{d}{d t} E_{\varepsilon}\left[\boldsymbol{u}_{\varepsilon}+t \boldsymbol{v}\right]\right|_{t=0}=\int_{\Omega}\left\{S_{t}(\right. & \left.,\left|\operatorname{curl} \boldsymbol{u}_{\varepsilon}\right|^{2}\right) \operatorname{curl} \boldsymbol{u}_{\varepsilon} \cdot \operatorname{curl} \boldsymbol{v} \\
& \left.+\frac{1}{\varepsilon} S_{t}\left(x,\left(\operatorname{div} \boldsymbol{u}_{\varepsilon}\right)^{2}\right)\left(\operatorname{div} \boldsymbol{u}_{\varepsilon}\right)(\operatorname{div} \boldsymbol{v})\right\} d x-\langle\boldsymbol{f}, \boldsymbol{v}\rangle=0 .
\end{aligned}
$$

Thus we obtain the following proposition.

Proposition 3.3. The minimizer $\boldsymbol{u}_{\varepsilon}$ in Proposition 3.2 is a unique weak solution of (3.1) in the sense of Definition 3.3.

Proof. It suffices to prove the uniqueness. Let $\boldsymbol{u}_{\varepsilon}^{1}, \boldsymbol{u}_{\varepsilon}^{2} \in \boldsymbol{W}_{0}^{1, p}(\Omega)$ be two weak solutions of (3.1). Taking $\boldsymbol{v}=\boldsymbol{u}_{\varepsilon}^{1}-\boldsymbol{u}_{\varepsilon}^{2}$ as a test function of (3.2), we have

$$
\begin{aligned}
\int_{\Omega}\{( & \left.\left.\left.S_{t}\left(x,\left|\operatorname{curl} \boldsymbol{u}_{\varepsilon}^{1}\right|^{2}\right) \operatorname{curl} \boldsymbol{u}_{\varepsilon}^{1}-\left.S_{t}\left(x, \mid \operatorname{curl} \boldsymbol{u}_{\varepsilon}^{2}\right)\right|^{2}\right) \operatorname{curl} \boldsymbol{u}_{\varepsilon}^{2}\right)\right) \cdot \operatorname{curl}\left(\boldsymbol{u}_{\varepsilon}^{1}-\boldsymbol{u}_{\varepsilon}^{2}\right) \\
& \left.+\frac{1}{\varepsilon}\left(S_{t}\left(x,\left(\operatorname{div} \boldsymbol{u}_{\varepsilon}^{1}\right)^{2}\right) \operatorname{div} \boldsymbol{u}_{\varepsilon}^{1}-S_{t}\left(x,\left(\operatorname{div} \boldsymbol{u}_{\varepsilon}^{2}\right)\right) \operatorname{div} \boldsymbol{u}_{\varepsilon}^{2}\right) \operatorname{div}\left(\boldsymbol{u}_{\varepsilon}^{1}-\boldsymbol{u}_{\varepsilon}^{2}\right)\right\} d x=0 .
\end{aligned}
$$

It follows from Lemma 2.2 that

$$
\int_{\Omega}\left(\left|\operatorname{curl}\left(\boldsymbol{u}_{\varepsilon}^{1}-\boldsymbol{u}_{\varepsilon}^{2}\right)\right|^{p}+\frac{1}{\varepsilon}\left|\operatorname{div}\left(\boldsymbol{u}_{\varepsilon}^{1}-\boldsymbol{u}_{\varepsilon}^{2}\right)\right|^{p} d x \leq 0 \text { if } p \geq 2,\right.
$$

and

$$
\begin{aligned}
\int_{\Omega}\left\{\left(\left|\operatorname{curl} \boldsymbol{u}_{\varepsilon}^{1}\right|\right.\right. & \left.+\left|\operatorname{curl} \boldsymbol{u}_{\varepsilon}^{2}\right|\right)^{p-2}\left|\operatorname{curl}\left(\boldsymbol{u}_{\varepsilon}^{1}-\boldsymbol{u}_{\varepsilon}^{2}\right)\right|^{2} \\
& \left.+\frac{1}{\varepsilon}\left(\left|\operatorname{div} \boldsymbol{u}_{\varepsilon}^{1}\right|+\left|\operatorname{div} \boldsymbol{u}_{\varepsilon}^{2}\right|\right)^{p-2}\left|\operatorname{div}\left(\boldsymbol{u}_{\varepsilon}^{1}-\boldsymbol{u}_{\varepsilon}^{2}\right)\right|^{2}\right\} d x \leq 0 \text { if } 1<p<2
\end{aligned}
$$

Therefore, we have

$$
\operatorname{curl}\left(\boldsymbol{u}_{\varepsilon}^{1}-\boldsymbol{u}_{\varepsilon}^{2}\right)=\mathbf{0} \text { and } \operatorname{div}\left(\boldsymbol{u}_{\varepsilon}^{1}-\boldsymbol{u}_{\varepsilon}^{2}\right)=0 \text { in } \Omega \text {. }
$$

By (3.4), we have $\boldsymbol{u}_{\varepsilon}^{1}=\boldsymbol{u}_{\varepsilon}^{2}$.

Remark 3.4. From this proposition, we can see that the minimizer of the minimization problem (3.3) is also unique.

Proof of Theorem 2.5 
Let $\boldsymbol{u}_{\varepsilon} \in \boldsymbol{W}_{0}^{1, p}(\Omega)$ be a unique minimizer of (3.3). Then $\boldsymbol{u}_{\varepsilon}$ is a weak solution of (3.1). If we take $\boldsymbol{v}=\boldsymbol{u}_{\varepsilon}$ as a test function of (3.2), then

$$
\begin{aligned}
\int_{\Omega}\left\{\left(S_{t}\left(x,\left|\operatorname{curl} \boldsymbol{u}_{\varepsilon}\right|^{2}\right)\left|\operatorname{curl} \boldsymbol{u}_{\varepsilon}\right|^{2}+\frac{1}{\varepsilon} S_{t}\left(x,\left(\operatorname{div} \boldsymbol{u}_{\varepsilon}\right)^{2}\right)\left(\operatorname{div} \boldsymbol{u}_{\varepsilon}\right)^{2}\right\} d x\right. & \\
& =\left\langle\boldsymbol{f}, \boldsymbol{u}_{\varepsilon}\right\rangle .
\end{aligned}
$$

Since $\frac{1}{\varepsilon} \geq 1$, we have

$$
\begin{aligned}
\lambda \int_{\Omega}\left(\left|\operatorname{curl} \boldsymbol{u}_{\varepsilon}\right|^{p}+\left|\operatorname{div} \boldsymbol{u}_{\varepsilon}\right|^{p}\right) d x \leq\|\boldsymbol{f}\|_{\boldsymbol{W}^{-1, p^{\prime}}(\Omega)} & \left\|\boldsymbol{u}_{\varepsilon}\right\|_{\boldsymbol{W}_{0}^{1, p}(\Omega)} \\
& \leq C(\delta)\|\boldsymbol{f}\|_{\boldsymbol{W}^{-1, p^{\prime}}(\Omega)}^{p^{\prime}}+\delta\left\|\boldsymbol{u}_{\varepsilon}\right\|_{\boldsymbol{W}_{0}^{1, p}(\Omega)}^{p}
\end{aligned}
$$

for any $\delta>0$. If we choose $\delta>0$ so that $\delta<\lambda / 2 C_{2}$, it follows from (3.4) that there exists a constant $C>0$ depending only on $p, \lambda$ and $\Omega$ such that

$$
\left\|\boldsymbol{u}_{\varepsilon}\right\|_{\boldsymbol{W}_{0}^{1, p}(\Omega)}^{p} \leq C\|\boldsymbol{f}\|_{\boldsymbol{W}^{-1, p^{\prime}}(\Omega)}^{p^{\prime}} .
$$

Moreover, from (3.7) and (3.6), we have

$$
\begin{aligned}
\lambda \int_{\Omega}\left|\operatorname{div} \boldsymbol{u}_{\varepsilon}\right|^{p} d x & \leq \int_{\Omega} S_{t}\left(x,\left(\operatorname{div} \boldsymbol{u}_{\varepsilon}\right)^{2}\right)\left(\operatorname{div} \boldsymbol{u}_{\varepsilon}\right)^{2} d x \\
& \leq \varepsilon\|\boldsymbol{f}\|_{\boldsymbol{W}^{-1, p^{\prime}}(\Omega)}\left\|\boldsymbol{u}_{\varepsilon}\right\|_{\boldsymbol{W}_{0}^{1, p}(\Omega)} \\
& \leq C^{1 / p} \varepsilon\|\boldsymbol{f}\|_{\boldsymbol{W}^{-1, p^{\prime}}(\Omega)}^{p^{\prime}}
\end{aligned}
$$

Hence, we have $\operatorname{div} \boldsymbol{u}_{\varepsilon} \rightarrow 0$ strongly in $L^{p}(\Omega)$ as $\varepsilon \rightarrow 0$. On the other hand, from (3.7), there exists a subsequence $\left\{\boldsymbol{u}_{\varepsilon_{j}}\right\}$ of $\left\{\boldsymbol{u}_{\varepsilon}\right\}$ such that $\boldsymbol{u}_{\varepsilon_{j}} \rightarrow \boldsymbol{u}$ weakly in $\boldsymbol{W}_{0}^{1, p}(\Omega)$. This implies $\operatorname{div} \boldsymbol{u}=0$ in $\Omega$. From (3.7), we have

$$
\|\boldsymbol{u}\|_{\boldsymbol{W}_{0}^{1, p}(\Omega)}^{p} \leq \liminf _{\varepsilon_{j} \rightarrow 0}\left\|\boldsymbol{u}_{\varepsilon_{j}}\right\|_{\boldsymbol{W}_{0}^{1, p}(\Omega)}^{p} \leq C\|\boldsymbol{f}\|_{\boldsymbol{W}^{-1, p^{\prime}}(\Omega)}^{p^{\prime}} .
$$

If we define $\pi_{\varepsilon}=-\frac{1}{\varepsilon} S_{t}\left(x,\left(\operatorname{div} \boldsymbol{u}_{\varepsilon}\right)^{2}\right) \operatorname{div} \boldsymbol{u}_{\varepsilon}$, then it follows from (3.8) and (2.1a) that

$$
\left\|\pi_{\varepsilon}\right\|_{L^{p^{\prime}(\Omega)}}^{p^{\prime}} \leq \frac{1}{\varepsilon} \Lambda \int_{\Omega}\left|\operatorname{div} \boldsymbol{u}_{\varepsilon}\right|^{p} d x \leq \frac{\Lambda C^{1 / p}}{\lambda}\|\boldsymbol{f}\|_{W^{-1, p^{\prime}}(\Omega)}^{p^{\prime}} .
$$

Passing to a subsequence, we may assume that $\pi_{\varepsilon_{j}} \rightarrow \pi$ weakly in $L^{p^{\prime}}(\Omega)$ and

$$
\|\pi\|_{L^{p^{\prime}(\Omega)}}^{p^{\prime}} \leq \liminf _{\varepsilon_{j} \rightarrow 0}\left\|\pi_{\varepsilon_{j}}\right\|_{L^{p^{\prime}}(\Omega)}^{p^{\prime}} \leq \frac{\Lambda C^{1 / p}}{\lambda}\|\boldsymbol{f}\|_{W^{-1, p^{\prime}}(\Omega)}^{p^{\prime}} .
$$

On the other hand, since

$$
\int_{\Omega}\left|S_{t}\left(x,\left|\operatorname{curl} \boldsymbol{u}_{\varepsilon}\right|^{2}\right) \operatorname{curl} \boldsymbol{u}_{\varepsilon}\right|^{p^{\prime}} d x \leq \Lambda^{p^{\prime}} \int_{\Omega}\left|\operatorname{curl} \boldsymbol{u}_{\varepsilon}\right|^{p} d x \leq \Lambda^{p^{\prime}} C\|\boldsymbol{f}\|_{W^{-1, p}(\Omega)}^{p^{\prime}},
$$


$\left\{S_{t}\left(x,\left|\operatorname{curl} \boldsymbol{u}_{\varepsilon}\right|^{2}\right) \operatorname{curl} \boldsymbol{u}_{\varepsilon}\right\}$ is bounded in $\boldsymbol{L}^{p^{\prime}}(\Omega)$. Passing to a subsequence, we may assume that

$$
S_{t}\left(x,\left|\operatorname{curl} \boldsymbol{u}_{\varepsilon_{j}}\right|^{2}\right) \operatorname{curl} \boldsymbol{u}_{\varepsilon_{j}} \rightarrow \boldsymbol{w} \text { weakly in } \boldsymbol{L}^{p^{\prime}}(\Omega) .
$$

Since

$$
\int_{\Omega}\left\{S_{t}\left(x,\left|\operatorname{curl} \boldsymbol{u}_{\varepsilon_{j}}\right|^{2} \operatorname{curl} \boldsymbol{u}_{\varepsilon_{j}} \cdot \operatorname{curl} \boldsymbol{v}-\pi_{\varepsilon_{j}} \operatorname{div} \boldsymbol{v}\right\} d x=\langle\boldsymbol{f}, \boldsymbol{v}\rangle\right.
$$

for all $\boldsymbol{v} \in \boldsymbol{W}_{0}^{1, p}(\Omega)$, letting $\varepsilon_{j} \rightarrow 0$, we have

$$
\int_{\Omega}(\boldsymbol{w} \cdot \operatorname{curl} \boldsymbol{v}-\pi \operatorname{div} \boldsymbol{v}) d x=\langle\boldsymbol{f}, \boldsymbol{v}\rangle \text { for all } \boldsymbol{v} \in \boldsymbol{W}_{0}^{1, p}(\Omega)
$$

In particular, since $\operatorname{div} \boldsymbol{u}=0$ in $\Omega$, we have

$$
\int_{\Omega} \boldsymbol{w} \cdot \operatorname{curl} \boldsymbol{u} d x=\langle\boldsymbol{f}, \boldsymbol{u}\rangle
$$

Since $\operatorname{div} \boldsymbol{u}_{\varepsilon_{j}} \rightarrow 0$ strongly in $L^{p}(\Omega)$, we have

$$
\begin{array}{r}
\lim _{j \rightarrow \infty} \int_{\Omega} S_{t}\left(x,\left|\operatorname{curl} \boldsymbol{u}_{\varepsilon_{j}}\right|^{2}\right)\left|\operatorname{curl} \boldsymbol{u}_{\varepsilon_{j}}\right|^{2} d x=\lim _{j \rightarrow \infty} \int_{\Omega} \pi_{\varepsilon_{j}} \operatorname{div} \boldsymbol{u}_{\varepsilon_{j}} d x+\left\langle\boldsymbol{f}, \boldsymbol{u}_{\varepsilon_{j}}\right\rangle \\
=\langle\boldsymbol{f}, \boldsymbol{u}\rangle=\int_{\Omega} \boldsymbol{w} \cdot \operatorname{curl} \boldsymbol{u} d x .
\end{array}
$$

By the monotonicity (Lemma 2.2), we have

$$
\begin{aligned}
\int_{\Omega} S_{t}\left(x,\left|\operatorname{curl} \boldsymbol{u}_{\varepsilon_{j}}\right|^{2}\right) \operatorname{curl} \boldsymbol{u}_{\varepsilon_{j}} \cdot \operatorname{curl}\left(\boldsymbol{u}_{\varepsilon_{j}}-\boldsymbol{v}\right) d x & \\
& -\int_{\Omega} S_{t}\left(x,|\operatorname{curl} \boldsymbol{v}|^{2}\right) \operatorname{curl} \boldsymbol{v} \cdot \operatorname{curl}\left(\boldsymbol{u}_{\varepsilon_{j}}-\boldsymbol{v}\right) d x \geq 0 .
\end{aligned}
$$

Letting $j \rightarrow \infty$, we have

$$
\int_{\Omega}\left(\boldsymbol{w}-S_{t}\left(x,|\operatorname{curl} \boldsymbol{v}|^{2}\right) \operatorname{curl} \boldsymbol{v}\right) \cdot \operatorname{curl}(\boldsymbol{u}-\boldsymbol{v}) d x \geq 0 \text { for all } \boldsymbol{v} \in \boldsymbol{W}_{0}^{1, p}(\Omega) .
$$

For any $\boldsymbol{\phi} \in \boldsymbol{W}_{0}^{1, p}(\Omega)$, put $\boldsymbol{v}=\boldsymbol{u}-\alpha \boldsymbol{\phi}(\alpha>0)$. Then we have

$$
\int_{\Omega}\left(\boldsymbol{w}-S_{t}\left(x,|\operatorname{curl} \boldsymbol{u}-\alpha \operatorname{curl} \phi|^{2}\right)(\operatorname{curl} \boldsymbol{u}-\alpha \operatorname{curl} \phi) \cdot \alpha \operatorname{curl} \phi d x \geq 0 .\right.
$$

If we divide this inequality by $\alpha$, and then let $\alpha \rightarrow 0$, we have

$$
\int_{\Omega}\left(\boldsymbol{w}-S_{t}\left(x,|\operatorname{curl} \boldsymbol{u}|^{2}\right) \operatorname{curl} \boldsymbol{u}\right) \cdot \operatorname{curl} \phi d x \geq 0
$$

for all $\phi \in \boldsymbol{W}_{0}^{1, p}(\Omega)$. This implies

$$
\int_{\Omega}\left(\boldsymbol{w}-S_{t}\left(x,|\operatorname{curl} \boldsymbol{u}|^{2}\right) \operatorname{curl} \boldsymbol{u}\right) \cdot \operatorname{curl} \phi d x=0
$$


for all $\phi \in \boldsymbol{W}_{0}^{1, p}(\Omega)$. Thus we have

$$
\left.\int_{\Omega}\left(S_{t}\left(x,|\operatorname{curl} \boldsymbol{u}|^{2}\right) \operatorname{curl} \boldsymbol{u} \cdot \operatorname{curl} \boldsymbol{v}-\pi \operatorname{div} \boldsymbol{v}\right)\right) d x=\langle\boldsymbol{f}, \boldsymbol{v}\rangle \text { for all } \boldsymbol{v} \in \boldsymbol{W}_{0}^{1, p}(\Omega) .
$$

Therefore, $(\boldsymbol{u}, \pi) \in \boldsymbol{W}_{0}^{1, p}(\Omega) \times L^{p}(\Omega)$ is a weak solution of (3.1).

Next we show the uniqueness of solution. Let $\left(\boldsymbol{u}_{1}, \pi_{1}\right),\left(\boldsymbol{u}_{2}, \pi_{2}\right) \in \boldsymbol{W}_{0}^{1, p}(\Omega) \times L^{p^{\prime}}(\Omega) / \mathbb{R}$ be two weak solutions of (3.1). If we take $\boldsymbol{v}=\boldsymbol{u}_{1}-\boldsymbol{u}_{2}$ as a test function of (3.2), then we have

$$
\int_{\Omega} S_{t}\left(x,\left|\operatorname{curl} \boldsymbol{u}_{i}\right|^{2}\right) \operatorname{curl} \boldsymbol{u}_{i} \cdot \operatorname{curl}\left(\boldsymbol{u}_{1}-\boldsymbol{u}_{2}\right) d x=\left\langle\boldsymbol{f}, \boldsymbol{u}_{1}-\boldsymbol{u}_{2}\right\rangle \text { for } i=1,2
$$

because of $\operatorname{div} \boldsymbol{u}_{i}=0$ in $\Omega$. Thus we have

$$
\int_{\Omega}\left(S_{t}\left(x,\left|\operatorname{curl} \boldsymbol{u}_{1}\right|^{2}\right) \operatorname{curl} \boldsymbol{u}_{1}-S_{t}\left(x,\left|\operatorname{curl} \boldsymbol{u}_{2}\right|^{2}\right) \operatorname{curl} \boldsymbol{u}_{2}\right) \cdot \operatorname{curl}\left(\boldsymbol{u}_{1}-\boldsymbol{u}_{2}\right) d x=0
$$

By the strict monotonicity (Lemma 2.2), we have $\operatorname{curl}\left(\boldsymbol{u}_{1}-\boldsymbol{u}_{2}\right)=\mathbf{0}$ and $\operatorname{div}\left(\boldsymbol{u}_{1}-\right.$ $\left.\boldsymbol{u}_{2}\right)=0$ in $\Omega$. By (3.4), we have $\boldsymbol{u}_{1}=\boldsymbol{u}_{2}$. From this, we have $\nabla\left(\pi_{1}-\pi_{2}\right)=\mathbf{0}$ in the distribution sense, so $\pi_{1}-\pi_{2}$ is a constant, i.e., $\pi_{1}=\pi_{2}$ in $L^{p^{\prime}}(\Omega) / \mathbb{R}$.

Finally we show the estimate (2.6). Taking $\boldsymbol{v}=\boldsymbol{u}$ as a test function of (2.5), since $\operatorname{div} \boldsymbol{u}=0$ in $\Omega$, we have

$$
\begin{aligned}
\lambda \int_{\Omega}|\operatorname{curl} \boldsymbol{u}|^{p} d x \leq \int_{\Omega} S_{t}\left(x,|\operatorname{curl} \boldsymbol{u}|^{2}\right)|\operatorname{curl} \boldsymbol{u}|^{2} d x & \\
& =\langle\boldsymbol{f}, \boldsymbol{u}\rangle \leq\|\boldsymbol{f}\|_{\boldsymbol{W}^{-1, p^{\prime}}(\Omega)}\|\boldsymbol{u}\|_{\boldsymbol{W}_{0}^{1, p}(\Omega)} .
\end{aligned}
$$

By the same arguments as above, we have

$$
\|\boldsymbol{u}\|_{\boldsymbol{W}_{0}^{1, p}(\Omega)}^{p} \leq C_{1}\|\boldsymbol{f}\|_{\boldsymbol{W}^{-1, p^{\prime}}(\Omega)}^{p^{\prime}} .
$$

If $c_{\pi}=\frac{1}{|\Omega|} \int_{\Omega} \pi d x$, we have

$$
\int_{\Omega}\left(\pi-c_{\pi}\right) \operatorname{div} \boldsymbol{v} d x=\int_{\Omega} \pi \operatorname{div} \boldsymbol{v} d x \text { for all } \boldsymbol{v} \in \boldsymbol{W}_{0}^{1, p}(\Omega)
$$

because of the fact $\int_{\Omega} \operatorname{div} \boldsymbol{v} d x=0$ from the divergence theorem. Therefore we may assume that $\pi \in L_{0}^{p^{\prime}}(\Omega)=\left\{\varphi \in L^{p^{\prime}}(\Omega) ; \int_{\Omega} \varphi d x=0\right\}$. For any $\phi \in L^{p}(\Omega)$, $\phi-c_{\phi} \in L_{0}^{p}(\Omega)$. According to Amrouche and Girault [1, Corollary 3.1], the operator $\operatorname{div}: \boldsymbol{W}_{0}^{1, p}(\Omega) / V^{1, p} \rightarrow L_{0}^{p}(\Omega)$ is isomorphism onto, where $V^{1, p}=\{\boldsymbol{v} \in$ $\boldsymbol{W}_{0}^{1, p}(\Omega) ; \operatorname{div} \boldsymbol{v}=0$ in $\left.\Omega\right\}$. Hence there exists $[\boldsymbol{w}] \in \boldsymbol{W}_{0}^{1, p}(\Omega) / V^{1, p}$ such that $\operatorname{div} \boldsymbol{w}=\phi-c_{\phi}$ and

$$
\|[\boldsymbol{w}]\|_{\boldsymbol{W}_{0}^{1, p}(\Omega) / V^{1, p}} \leq C\left\|\phi-c_{\phi}\right\|_{L^{p}(\Omega)} \leq C_{1}\|\phi\|_{L^{p}(\Omega)} .
$$


We claim that $\|[\boldsymbol{w}]\|_{\boldsymbol{W}_{0}^{1, p}(\Omega)}=\inf _{\boldsymbol{v} \in V^{1, p}}\|\boldsymbol{w}+\boldsymbol{v}\|_{\boldsymbol{W}_{0}^{1, p}(\Omega)}$ is achieved. In fact, $\left\{\boldsymbol{v}_{j}\right\} \subset$ $V^{1, p}$ be a minimizing sequence of $\|[\boldsymbol{w}]\|_{\boldsymbol{W}_{0}^{1, p}(\Omega) / V^{1, p}}$. Then

$$
\left\|\boldsymbol{w}+\boldsymbol{v}_{j}\right\|_{\boldsymbol{W}_{0}^{1, p}(\Omega)}=\|[\boldsymbol{w}]\|_{\boldsymbol{W}_{0}^{1, p}(\Omega) / V^{1, p}}+o(1) \text { as } j \rightarrow \infty .
$$

Then $\left\{\boldsymbol{v}_{j}\right\}$ is bounded in $\boldsymbol{W}_{0}^{1, p}(\Omega)$. Passing to a subsequence, we may assume that $\boldsymbol{v}_{j} \rightarrow \boldsymbol{v}_{0}$ weakly in $\boldsymbol{W}_{0}^{1, p}(\Omega)$, so $\operatorname{div} \boldsymbol{v}_{0}=0$ in $\Omega$, i.e,, $\boldsymbol{v}_{0} \in V^{1, p}$. Moreover, we have

$$
\left\|\boldsymbol{w}+\boldsymbol{v}_{0}\right\|_{\boldsymbol{W}_{0}^{1, p}(\Omega)} \leq \liminf _{j \rightarrow \infty}\left\|\boldsymbol{w}+\boldsymbol{v}_{j}\right\|_{\boldsymbol{W}_{0}^{1, p}(\Omega)}=\|[\boldsymbol{w}]\|_{\boldsymbol{W}_{0}^{1, p}(\Omega) / V^{1, p}} .
$$

Thus we have $\operatorname{div}\left(\boldsymbol{w}+\boldsymbol{v}_{0}\right)=\operatorname{div} \boldsymbol{w}=\phi-c_{\phi}$. Hence we can assume that $\operatorname{div} \boldsymbol{w}=\phi-c_{\phi}$ and

$$
\|\boldsymbol{w}\|_{\boldsymbol{W}_{0}^{1, p}(\Omega)} \leq C\|\phi\|_{L^{p}(\Omega)} .
$$

Taking $\boldsymbol{v}=\boldsymbol{w}$ as a test function of (2.5), since $\pi \in L_{0}^{p^{\prime}}(\Omega)$ and satisfies $\int_{\Omega} \pi c_{\phi} d x=0$, we have

$$
\int_{\Omega} \pi \phi d x=\int_{\Omega} S_{t}\left(x,|\operatorname{curl} \boldsymbol{u}|^{2}\right) \operatorname{curl} \boldsymbol{u} \cdot \operatorname{curl} \boldsymbol{w} d x-\langle\boldsymbol{f}, \boldsymbol{w}\rangle .
$$

Therefore, by the Hölder inequality and (3.14), we have

$$
\begin{aligned}
\left|\int_{\Omega} \pi \phi d x\right| & \leq \Lambda \int_{\Omega}|\operatorname{curl} \boldsymbol{u}|^{p-1}|\operatorname{curl} \boldsymbol{w}| d x+\|\boldsymbol{f}\|_{\boldsymbol{W}^{-1, p^{\prime}(\Omega)}}\|\boldsymbol{w}\|_{\boldsymbol{W}_{0}^{1, p}(\Omega)} \\
& \leq \Lambda\|\operatorname{curl} \boldsymbol{u}\|_{\boldsymbol{L}^{p}(\Omega)}^{p-1}\|\operatorname{curl} \boldsymbol{w}\|_{\boldsymbol{L}^{p}(\Omega)}+\|\boldsymbol{f}\|_{\boldsymbol{W}^{-1, p^{\prime}}(\Omega)}\|\boldsymbol{w}\|_{\boldsymbol{W}_{0}^{1, p}(\Omega)} \\
& \leq C \Lambda\left(\|\boldsymbol{u}\|_{\boldsymbol{W}_{0}^{1, p}(\Omega)}^{p-1}+\|\boldsymbol{f}\|_{\boldsymbol{W}^{-1, p^{\prime}}(\Omega)}\|\phi\|_{L^{p}(\Omega)}\right. \\
& \leq C\|\boldsymbol{f}\|_{\boldsymbol{W}^{-1, p^{\prime}}(\Omega)}\|\phi\|_{L^{p}(\Omega)} .
\end{aligned}
$$

for all $\phi \in L^{p}(\Omega)$. Hence we have

$$
\|\pi\|_{L^{p^{\prime}}(\Omega)} \leq C\|\boldsymbol{f}\|_{\boldsymbol{W}^{-1, p^{\prime}}(\Omega)} .
$$

Summing up (3.14) and (3.16), we get the estimate (2.6). This completes the proof of Theorem 2.5.

\section{CONTINUOUS DEPENDENCE OF A WEAK SOLUTION ON THE DATA}

In this section, we consult the continuous dependence of a weak solution of (2.4) on the data. In order to do so, for every $n=0,1, \ldots$, let $S^{(n)}(x, t)$ satisfy (2.1a)-(2.1c) with the same constants $\lambda$ and $\Lambda$, and let $\boldsymbol{f}_{n} \in \boldsymbol{W}^{-1, p^{\prime}}(\Omega)$. For $n=0,1, \ldots$, assume that $\left(\boldsymbol{u}_{n}, \pi_{n}\right) \in \boldsymbol{W}_{0}^{1, p}(\Omega) \times L^{p^{\prime}}(\Omega) / \mathbb{R}$ is weak solution of (2.4), i.e.,

$$
\begin{cases}\operatorname{curl}\left[S_{t}^{(n)}\left(x,\left|\operatorname{curl} \boldsymbol{u}_{n}\right|^{2}\right) \operatorname{curl} \boldsymbol{u}_{n}\right]+\nabla \pi_{n}=\boldsymbol{f}_{n} & \text { in } \Omega, \\ \operatorname{div} \boldsymbol{u}_{n}=0 & \text { in } \Omega, \\ \boldsymbol{u}_{n}=\mathbf{0} & \text { on } \Gamma .\end{cases}
$$


More precisely, $\left(\boldsymbol{u}_{n}, \pi_{n}\right)$ satisfies

$$
\begin{aligned}
\int_{\Omega} S_{t}^{(n)}\left(x,\left|\operatorname{curl} \boldsymbol{u}_{n}\right|^{2}\right) \operatorname{curl} \boldsymbol{u}_{n} \cdot & \operatorname{curl} \boldsymbol{v} d x-\int_{\Omega} \pi_{n} \operatorname{div} \boldsymbol{v} d x \\
& =\left\langle\boldsymbol{f}_{n}, \boldsymbol{v}\right\rangle \text { for all } \boldsymbol{v} \in \boldsymbol{W}_{0}^{1, p}(\Omega)(n=0,1, \ldots) .
\end{aligned}
$$

Then we have the following theorem on the continuous dependence on the data.

Theorem 4.1. We assume that for every $n=0,1, \ldots, a$ Carathéodory function $S^{(n)}(x, t)$ satisfies (2.1a)-(2.1c) with the same constants $\lambda$ and $\Lambda$, and $\boldsymbol{f}_{n} \in \boldsymbol{W}^{-1, p^{\prime}}(\Omega)$. Let $\left(\boldsymbol{u}_{n}, \pi_{n}\right) \in \boldsymbol{W}_{0}^{1, p}(\Omega) \times L^{p^{\prime}}(\Omega) / \mathbb{R}$ be a unique weak solution of $(2.4)$. If $S_{t}^{(n)}(x, t) \rightarrow$ $S_{t}^{(0)}(x, t)$ a.e. in $\Omega \times[0, \infty)$ and $\boldsymbol{f}_{n} \rightarrow \boldsymbol{f}_{0}$ in $\boldsymbol{W}^{-1, p^{\prime}}(\Omega)$ as $n \rightarrow \infty$, then $\boldsymbol{u}_{n} \rightarrow \boldsymbol{u}_{0}$ in $\boldsymbol{W}_{0}^{1, p}(\Omega)$ and $\pi_{n} \rightarrow \pi_{0}$ in $L^{p^{\prime}}(\Omega) / \mathbb{R}$ as $n \rightarrow \infty$.

In particular case where $S^{(n)}(x, t)=S^{(0)}(x, t)$ for all $n=1, \ldots$, there exists a constant $C>0$ depending only on $p, \lambda, \Lambda, \Omega$ and $\left\|\boldsymbol{f}_{0}\right\|_{\boldsymbol{W}^{-1, p^{\prime}(\Omega)}}$ such that

$$
\begin{aligned}
\left\|\boldsymbol{u}_{n}-\boldsymbol{u}_{0}\right\|_{\boldsymbol{W}_{0}^{1, p}(\Omega)}^{p \vee p^{\prime}}+\left\|\pi_{n}-\pi_{0}\right\|_{L^{p}(\Omega) / \mathbb{R}}^{p \vee p^{\prime}} & \\
& \leq C\left(\left\|\boldsymbol{f}_{n}-\boldsymbol{f}_{0}\right\|_{\boldsymbol{W}^{-1, p^{\prime}}(\Omega)}^{p^{\prime}}+\left\|\boldsymbol{f}_{n}-\boldsymbol{f}_{0}\right\|_{\boldsymbol{W}^{-1, p^{\prime}}(\Omega)}^{p}\right),
\end{aligned}
$$

where $p \vee p^{\prime}=\max \left\{p, p^{\prime}\right\}$.

Proof. Taking $\boldsymbol{v}=\boldsymbol{u}_{n}-\boldsymbol{u}_{0}$ as a test function of (4.2), since $\operatorname{div}\left(\boldsymbol{u}_{n}-\boldsymbol{u}_{0}\right)=0$ in $\Omega$, we have

$$
\begin{gathered}
\int_{\Omega}\left(S_{t}^{(n)}\left(x,\left|\operatorname{curl} \boldsymbol{u}_{n}\right|^{2}\right) \operatorname{curl} \boldsymbol{u}_{n}-S_{t}^{(n)}\left(x,\left|\operatorname{curl} \boldsymbol{u}_{0}\right|^{2}\right) \operatorname{curl} \boldsymbol{u}_{0}\right) \cdot \operatorname{curl}\left(\boldsymbol{u}_{n}-\boldsymbol{u}_{0}\right) d x \\
=\left\langle\boldsymbol{f}_{n}-\boldsymbol{f}_{0}, \boldsymbol{u}_{n}-\boldsymbol{u}_{0}\right\rangle \\
-\int_{\Omega}\left(S_{t}^{(n)}\left(x,\left|\operatorname{curl} \boldsymbol{u}_{0}\right|^{2}\right) \operatorname{curl} \boldsymbol{u}_{0}-S_{t}^{(0)}\left(x,\left|\operatorname{curl} \boldsymbol{u}_{0}\right|^{2}\right) \operatorname{curl} \boldsymbol{u}_{0}\right) \cdot \operatorname{curl}\left(\boldsymbol{u}_{n}-\boldsymbol{u}_{0}\right) d x .
\end{gathered}
$$

Hereafter, for the brevity of notations, we put

$$
\begin{aligned}
F_{n}(k)=\| \boldsymbol{f}_{n}- & \boldsymbol{f}_{0} \|_{\boldsymbol{W}^{-1, p^{\prime}}(\Omega)}^{k} \\
& +\left\|S_{t}^{(n)}\left(x,\left|\operatorname{curl} \boldsymbol{u}_{0}\right|^{2}\right) \operatorname{curl} \boldsymbol{u}_{0}-S_{t}^{(0)}\left(x,\left|\operatorname{curl} \boldsymbol{u}_{0}\right|^{2}\right) \operatorname{curl} \boldsymbol{u}_{0}\right\|_{\boldsymbol{L}^{p^{\prime}}(\Omega)}^{k},
\end{aligned}
$$

and we denote constants depending only on $p, \lambda, \Lambda, \Omega$ and the constants in Lemma 2.2, 2.3 by $C, C_{1}, C_{2}, \ldots$ which may vary from line to line.

We estimate (4.3). 
When $p \geq 2$, it follows from Lemma 2.2 (ii) and the Young inequality that we have

$$
\begin{aligned}
& c_{2} \int_{\Omega}\left|\operatorname{curl}\left(\boldsymbol{u}_{n}-\boldsymbol{u}_{0}\right)\right|^{p} d x \\
& \quad \leq\left\|\boldsymbol{f}_{n}-\boldsymbol{f}_{0}\right\|_{\boldsymbol{W}^{-1, p^{\prime}}(\Omega)}\left\|\boldsymbol{u}_{n}-\boldsymbol{u}_{0}\right\|_{\boldsymbol{W}_{0}^{1, p}(\Omega)} \\
& \quad+\left\|S_{t}^{(n)}\left(x,\left|\operatorname{curl} \boldsymbol{u}_{0}\right|^{2}\right) \operatorname{curl} \boldsymbol{u}_{0}-S_{t}^{(0)}\left(x,\left|\operatorname{curl} \boldsymbol{u}_{0}\right|^{2}\right) \operatorname{curl} \boldsymbol{u}_{0}\right\|_{\boldsymbol{L}^{p^{\prime}}(\Omega)} \\
& \quad \times \| \operatorname{curl}\left(\boldsymbol{u}_{n}-\boldsymbol{u}_{0} \|_{\boldsymbol{L}^{p}(\Omega)}\right. \\
& \quad \leq C(\varepsilon) F_{n}\left(p^{\prime}\right)+\varepsilon\left\|\boldsymbol{u}_{n}-\boldsymbol{u}_{0}\right\|_{\boldsymbol{W}_{0}^{1, p}(\Omega)}^{p}
\end{aligned}
$$

for any $\varepsilon>0$. On the other hand, from (3.4),

$$
c_{2}\left\|\boldsymbol{u}_{n}-\boldsymbol{u}_{0}\right\|_{W_{0}^{1, p}(\Omega)}^{p} \leq c_{2} C_{2}\left\|\operatorname{curl}\left(\boldsymbol{u}_{n}-\boldsymbol{u}_{0}\right)\right\|_{L^{p}(\Omega)}^{p} .
$$

If we choose $\varepsilon>0$ so that $C_{2} \varepsilon=c_{2} / 2$, we have

$$
\left\|\boldsymbol{u}_{n}-\boldsymbol{u}_{0}\right\|_{\boldsymbol{W}_{0}^{1, p}(\Omega)}^{p} \leq C_{2} F_{n}\left(p^{\prime}\right)
$$

When $1<p<2$, using Lemma 2.2 (ii) to (4.3), we have

$$
\int_{\Omega}\left(\left|\operatorname{curl} \boldsymbol{u}_{n}\right|+\left|\operatorname{curl} \boldsymbol{u}_{0}\right|\right)^{p-2}\left|\operatorname{curl}\left(\boldsymbol{u}_{n}-\boldsymbol{u}_{0}\right)\right|^{2} d x \leq C F_{n}(1)\left\|\boldsymbol{u}_{n}-\boldsymbol{u}_{0}\right\|_{W_{0}^{1, p}(\Omega)} .
$$

Here if we use the reverse Hölder inequality (cf. Sobolev [10, p. 8]) with $0<s=$ $p / 2<1$ and $s^{\prime}=p /(p-2)$, then there exists a constant $c>0$ such that

$$
\begin{aligned}
\int_{\Omega}\left(\left|\operatorname{curl} \boldsymbol{u}_{n}\right|\right. & \left.+\left|\operatorname{curl} \boldsymbol{u}_{0}\right|\right)^{p-2}\left|\operatorname{curl}\left(\boldsymbol{u}_{n}-\boldsymbol{u}_{0}\right)\right|^{2} d x \\
& \geq c\left(\left\|\operatorname{curl} \boldsymbol{u}_{n}\right\|_{L^{p}(\Omega)}^{p}+\left\|\operatorname{curl} \boldsymbol{u}_{0}\right\|_{\boldsymbol{L}^{p}(\Omega)}^{p}\right)^{(p-2) / 2}\left\|\operatorname{curl}\left(\boldsymbol{u}_{n}-\boldsymbol{u}_{0}\right)\right\|_{\boldsymbol{L}^{p}(\Omega)}^{2} .
\end{aligned}
$$

Hence, it follows from (3.9) that

$$
\begin{aligned}
& \left\|\boldsymbol{u}_{n}-\boldsymbol{u}_{0}\right\|_{\boldsymbol{W}_{0}^{1, p}(\Omega)}^{2} \\
& \quad \leq C\left(\left\|\boldsymbol{f}_{n}\right\|_{\boldsymbol{W}^{-1, p^{\prime}}(\Omega)}^{p^{\prime}}+\left\|\boldsymbol{f}_{0}\right\|_{\boldsymbol{W}^{-1, p^{\prime}}(\Omega)}^{p^{\prime}}\right)^{(2-p) / 2} F_{n}(1)\left\|\boldsymbol{u}_{n}-\boldsymbol{u}_{0}\right\|_{\boldsymbol{W}_{0}^{1, p}(\Omega)} .
\end{aligned}
$$

Since we may assume that

$$
\left\|\boldsymbol{f}_{n}\right\|_{\boldsymbol{W}^{-1, p^{\prime}}(\Omega)}^{p^{\prime}} \leq C\left(\left\|\boldsymbol{f}_{0}\right\|_{\boldsymbol{W}^{-1, p^{\prime}}(\Omega)}^{p^{\prime}}+1\right)
$$

under the hypothesis, we can write

$$
\left\|\boldsymbol{u}_{n}-\boldsymbol{u}_{0}\right\|_{\boldsymbol{W}_{0}^{1, p}(\Omega)} \leq C_{2} F_{n}(1)
$$


We may assume that $\pi_{n}-\pi_{0} \in L_{0}^{p^{\prime}}(\Omega)$, so for any $\phi \in L^{p}(\Omega)$,

$$
\int_{\Omega}\left(\pi_{n}-\pi_{0}\right)\left(\phi-c_{\phi}\right) d x=\int_{\Omega}\left(\pi_{n}-\pi_{0}\right) \phi d x
$$

There exists $\boldsymbol{w} \in \boldsymbol{W}_{0}^{1, p}(\Omega)$ such that $\operatorname{div} \boldsymbol{w}=\phi-c_{\phi}$ (cf. [1, Corollary 3.1]), and

$$
\|\boldsymbol{w}\|_{\boldsymbol{W}_{0}^{1, p}(\Omega)} \leq C\|\phi\|_{L^{p}(\Omega)} .
$$

Taking $\boldsymbol{v}=\boldsymbol{w}$ as a test function of (4.2), we have

$$
\begin{aligned}
\int_{\Omega}\left(S_{t}^{(n)}\left(x,\left|\operatorname{curl} \boldsymbol{u}_{n}\right|^{2}\right) \operatorname{curl} \boldsymbol{u}_{n}-S_{t}^{(0)}\left(x,\left|\operatorname{curl} \boldsymbol{u}_{0}\right|^{2}\right) \operatorname{curl} \boldsymbol{u}_{0}\right) \cdot \operatorname{curl} \boldsymbol{w} d x & \\
& -\int_{\Omega}\left(\pi_{n}-\pi_{0}\right) \phi d x=\left\langle\boldsymbol{f}_{n}-\boldsymbol{f}_{0}, \boldsymbol{w}\right\rangle .
\end{aligned}
$$

We write this equality in the following form.

$$
\int_{\Omega}\left(\pi_{n}-\pi_{0}\right) \phi d x=I_{1}+I_{2}-\left\langle\boldsymbol{f}_{n}-\boldsymbol{f}_{0}, \boldsymbol{w}\right\rangle
$$

where

$$
\begin{aligned}
& I_{1}=\int_{\Omega}\left(S_{t}^{(n)}\left(x,\left|\operatorname{curl} \boldsymbol{u}_{n}\right|^{2}\right) \operatorname{curl} \boldsymbol{u}_{n}-S_{t}^{(n)}\left(x,\left|\operatorname{curl} \boldsymbol{u}_{0}\right|^{2}\right) \operatorname{curl} \boldsymbol{u}_{0}\right) \cdot \operatorname{curl} \boldsymbol{w} d x, \\
& I_{2}=\int_{\Omega}\left(S_{t}^{(n)}\left(x,\left|\operatorname{curl} \boldsymbol{u}_{0}\right|^{2}\right) \operatorname{curl} \boldsymbol{u}_{0}-S_{t}^{(0)}\left(x,\left|\operatorname{curl} \boldsymbol{u}_{0}\right|^{2}\right) \operatorname{curl} \boldsymbol{u}_{0}\right) \cdot \operatorname{curl} \boldsymbol{w} d x .
\end{aligned}
$$

From (4.6) and the Hölder inequality, we have

$$
\begin{aligned}
&\left|I_{2}\right| \leq C\left\|S_{t}^{(n)}\left(x,\left|\operatorname{curl} \boldsymbol{u}_{0}\right|^{2}\right) \operatorname{curl} \boldsymbol{u}_{0}-S_{t}^{(0)}\left(x,\left|\operatorname{curl} \boldsymbol{u}_{0}\right|^{2}\right) \operatorname{curl} \boldsymbol{u}_{0}\right\|_{\boldsymbol{L}^{p^{\prime}}(\Omega)} \\
& \times\|\boldsymbol{w}\|_{\boldsymbol{W}_{0}^{1, p}(\Omega)} .
\end{aligned}
$$

We now estimate $I_{1}$. When $1<p<2$, using Lemma 2.3, (4.5) and (4.6), we have

$$
\begin{aligned}
\left|I_{1}\right| & \leq C_{4} \int_{\Omega}\left|\operatorname{curl}\left(\boldsymbol{u}_{n}-\boldsymbol{u}_{0}\right)\right|^{p-1}|\operatorname{curl} \boldsymbol{w}| d x \\
& \leq C_{4}\left\|\boldsymbol{u}_{n}-\boldsymbol{u}_{0}\right\|_{\boldsymbol{W}_{0}^{1, p}(\Omega)}^{p-1}\|\boldsymbol{w}\|_{\boldsymbol{W}_{0}^{1, p}(\Omega)} \\
& \leq C_{5} F_{n}(p-1)\|\phi\|_{L^{p}(\Omega)} .
\end{aligned}
$$

When $p \geq 2$, using Lemma 2.3, (4.4), (4.6), (2.6) and the Hölder inequality, we have

$$
\begin{aligned}
\left|I_{1}\right| \leq & \left.C_{1} \int_{\Omega}\left(\left|\operatorname{curl} \boldsymbol{u}_{n}\right|+\mid \operatorname{curl} \boldsymbol{u}_{0}\right) \mid\right)^{p-2}\left|\operatorname{curl}\left(\boldsymbol{u}_{n}-\boldsymbol{u}_{0}\right)\right||\operatorname{curl} \boldsymbol{w}| d x \\
\leq & C_{2}\left(\left\|\operatorname{curl} \boldsymbol{u}_{n}\right\|_{\boldsymbol{L}^{p}(\Omega)}+\left\|\operatorname{curl} \boldsymbol{u}_{0}\right\|_{\boldsymbol{L}^{p}(\Omega)}\right)^{p-2} \\
& \quad \times\left\|\operatorname{curl}\left(\boldsymbol{u}_{n}-\boldsymbol{u}_{0}\right)\right\| \boldsymbol{L}_{\boldsymbol{L}^{p}(\Omega)}\|\operatorname{curl} \boldsymbol{w}\|_{\boldsymbol{L}^{p}(\Omega)} \\
\leq & C_{3}\left(\left\|\boldsymbol{f}_{n}\right\|_{\boldsymbol{W}^{-1, p^{\prime}(\Omega)}}^{p^{\prime}-1}+\left\|\boldsymbol{f}_{0}\right\|_{\boldsymbol{W}^{-1, p^{\prime}(\Omega)}}^{p^{\prime}-1}\right)^{p-2} F_{n}\left(p^{\prime}-1\right)\|\phi\|_{L^{p}(\Omega)} .
\end{aligned}
$$


Therefore, we have

$$
\left\|\pi_{n}-\pi\right\|_{L^{p^{\prime}}(\Omega)} \leq \begin{cases}C_{1} F_{n}(1)+C_{2} F_{n}(p-1) & \text { if } 1<p<2 \\ C_{1} F_{n}(1)+C_{2} F_{n}\left(p^{\prime}-1\right) & \text { if } p \geq 2\end{cases}
$$

Hence we have

$$
\left\|\boldsymbol{u}_{n}-\boldsymbol{u}_{0}\right\|_{\boldsymbol{W}_{0}^{1, p}(\Omega)}^{p \vee p^{\prime}}+\left\|\pi_{n}-\pi_{0}\right\|_{L^{p^{\prime}}(\Omega)}^{p \vee p^{\prime}} \leq C\left(F_{n}(p)+F_{n}\left(p^{\prime}\right)\right) .
$$

Finally, we claim that $F_{n}(k) \rightarrow 0$ as $n \rightarrow \infty$, if $\boldsymbol{f}_{n} \rightarrow \boldsymbol{f}_{0}$ in $\boldsymbol{W}^{-1, p^{\prime}}(\Omega)$ and $S_{t}^{(n)}(x, t) \rightarrow S_{t}^{(0)}(x, t)$ a.e. $(x, t) \in \Omega \times[0, \infty)$.

In fact, from (2.1a), we have

$$
\begin{aligned}
& \left|S_{t}^{(n)}\left(x,\left|\operatorname{curl} \boldsymbol{u}_{0}\right|^{2}\right) \operatorname{curl} \boldsymbol{u}_{0}-S_{t}^{(0)}\left(x,\left|\operatorname{curl} \boldsymbol{u}_{0}\right|^{2}\right) \operatorname{curl} \boldsymbol{u}_{0}\right|^{p^{\prime}} \\
& \quad \leq(2 \Lambda)^{p^{\prime}}\left|\operatorname{curl} \boldsymbol{u}_{0}\right|^{p} \in L^{1}(\Omega)
\end{aligned}
$$

and $S_{t}^{(n)}\left(x,\left|\operatorname{curl} \boldsymbol{u}_{0}\right|^{2}\right) \operatorname{curl} \boldsymbol{u}_{0}-S_{t}^{0}\left(x,\left|\operatorname{curl} \boldsymbol{u}_{0}\right|^{2}\right) \operatorname{curl} \boldsymbol{u}_{0} \rightarrow 0$ a.e. in $\Omega$, so it follows from the Lebesgue dominated theorem that

$$
\left\|S_{t}^{(n)}\left(x,\left|\operatorname{curl} \boldsymbol{u}_{0}\right|^{2}\right) \operatorname{curl} \boldsymbol{u}_{0}-S_{t}^{(0)}\left(x,\left|\operatorname{curl} \boldsymbol{u}_{0}\right|^{2}\right) \operatorname{curl} \boldsymbol{u}_{0}\right\|_{\boldsymbol{L}^{p^{\prime}}(\Omega)} \rightarrow 0
$$

as $n \rightarrow \infty$. In the particular case, since $F_{n}(k)=\left\|\boldsymbol{f}_{n}-\boldsymbol{f}_{0}\right\|_{W^{-1, p^{\prime}}(\Omega)}^{k}$, the estimate is clear.

\section{REFERENCES}

[1] Amrouche, C., and V. Girault, V., 1994, "Decomposition of vector spaces and application to the Stokes problem in arbitrary dimension," Czechslovak Math. J., 44(1), pp. 109-140.

[2] Amrouche, C, and Seloula, N. H., 2013, " $L^{p}$-theory for vector potentials and Sobolev inequalities for vector fields: Application to the Stokes equations with pressure boundary conditions", Math. Models and Methods in Appl. Sci., 23(4), pp. 37-92.

[3] Aramaki, J., 2018, "Existence of weak solution for a class of abstract coupling system associated with stationary electromagnetic system", Taiwanese J. Math., 22(3), pp. 741-765.

[4] Aramaki, J., 2018, "Variational problem involving operator curl associated with p-curl system", Turkish J. Math., 42(3), pp. 949-966. 
[5] Aramaki, J., 2019, "Existence and regularity of a weak solution to a class of systems in a multi-connected domain”, J. Part. Diff. Eq., 32(1), pp. 1-19.

[6] Dautray, R., and Lions, J. L., 1988, Analyse MathÉmatique et Calsul Numérique pour les Sciences et les Technique, vol. 3 and vol. 7, Masson.

[7] Girault, V., and Raviart, P. A., 1986, Finite Element Method for Navier-Stokes Equations, Springer Series SCM.

[8] Jost, J., 2005, Postmodern Analysis: 3rd edition, Springer-Verlag, Berlin, Heidelberg.

[9] Temam, R., 1985, Navier-Stokes Equations. Theory and Analysis, North-Holland, Amsterdam.

[10] Sobolev, S., 1963, Applications of Functional Analysis in Mathematical Physics, Translation of Mathematical Monographs, Vol. 7, AMS, Providence, R. I.. 